\title{
Chemerin and the recruitment of NK cells to diseased skin ${ }^{\star}$
}

\author{
Joanna Skrzeczyńska-Moncznik ${ }^{1}$, Anna Stefańska ${ }^{1}$, Brian A. Zabel ${ }^{2,3}$, \\ Monika Kapińska-Mrowiecka ${ }^{4}$, Eugene C. Butcher ${ }^{2,3}$ and Joanna Cichy ${ }^{1 凶}$ \\ ${ }^{1}$ Department of Immunology, Faculty of Biochemistry, Biophysics and Biotechnology, Jagiellonian University, \\ Kraków, Poland; ${ }^{2}$ Stanford University Medical School, Stanford, USA; ${ }^{3}$ Veterans Affairs Palo Alto Health Care \\ System, Palo Alto, USA; ${ }^{4}$ Department of Dermatology, Żeromski Hospital, Kraków, Poland
}

Received: 07 April, 2009; revised: 29 May, 2009; accepted: 17 June, 2009

available on-line: 18 June, 2009

\begin{abstract}
Natural killer (NK) cells play a major role in the initial control of many viral pathogens and in the rejection of tumors. Consistent with their roles as immune sentinels, NK cells are found in inflamed skin, including lichen planus, psoriasis and atopic dermatitis (AD) lesions. In oral lichen planus lesions, the recruitment as well as intradermal colocalization of NK cells and pDC (plasmacytoid dendritic cells) appear to be mediated by chemerin, a recently identified protein ligand for chemokine-like receptor 1 (CMKLR1), a chemoattractant receptor expressed by both cell types. Dendritic cells can regulate NK cell activity, and NK cells can regulate DC-mediated responses. Since chemerin was recently implicated in recruitment of pDC to psoriatic skin, in this work we determined whether chemerin facilitates interactions between NK and pDC in psoriatic plaques through controlling influx of NK cells to diseased skin. We demonstrate that circulating NK cells from normal donors as well as psoriasis and AD patients respond similarly in functional migration assays to chemerin. However, differences in the distribution of NK cells and pDC in skin lesions suggest that recruitment of both NK cells and pDC is unlikely to be controlled solely by chemerin.
\end{abstract}

Keywords: chemerin, NK cells, skin diseases, viral pathogens

\section{INTRODUCTION}

Chemerin was initially named tazarotene-induced gene 2 (tig-2), a designation that reflects its transcriptional upregulation in lesional psoriatic skin by the anti-psoriatic synthetic retinoid tazarotene (Nagpal et al., 1997). More recently, through G protein-associated receptor screening assays, chemerin was identified as a protein ligand for the orphan seven-pass transmembrane receptor chemokine-like receptor 1 (CMKLR1) (Wittamer et al., 2003; Zabel et al., 2005b). Several specific functions have been ascribed to chemerin so far, including regulation of specific immune cell migration (Wittamer et al., 2003; Zabel et al., 2005b), anti-inflammatory effects on macrophages (Cash et al., 2008), and regula- tion of adipogenesis (Goralski et al., 2007). Human chemerin is synthesized as an aprox. $18 \mathrm{kDa}$ precursor which undergoes protease-mediated C-terminal truncation to generate a potent chemoattractant for CMKLR1-expressing cells. Enzymes that contribute to this conversion include host serine proteases of the coagulation, fibrinolytic and inflammatory cascades (Du et al., 2009; Wittamer et al., 2005; Zabel et al., 2005a), circulating carboxypeptidases (Du et al., 2009), as well as staphopain B, a cysteine protease secreted by Staphylococcus aureus (Kulig et al., 2007).

CMKLR1 exhibits a selective expression pattern among immune cells. The best documented is the presence of this receptor on immature plasmacytoid dendritic cells (pDC) and resident macrophages (Zabel et al., 2006a; Zabel et al., 2005b). In addition,

\footnotetext{
${ }^{\square}$ Corresponding author: Joanna Cichy, Department of Immunology, Faculty of Biochemistry, Biophysics and Biotechnology, Jagiellonian University, Kraków, Poland; e-mail: Joanna.Cichy@uj.edu.pl

* Presented at the XXXVI Winter School "Molecule interactions in health and disease" organized by the Faculty of Biochemistry, Biophysics and Biotechnology, Jagiellonian University, 21-26 February, 2009, Zakopane, Poland.

Abbreviations: AD, atopic dermatitis; CMKLR1, chemokine-like receptor 1; NK, natural killer; pDC, plasmacytoid dendritic cells; SspB, StaphopainB.
} 
recent data indicate that a subset of natural killer (NK) cells express functional CMKLR1 (Parolini et al., 2007). NK cells represent an important component of the immune system through their ability to both lyse target cells and to secrete a variety of immunoregulatory cytokines. Although NK cells are mainly recognized as cells of the innate immune system capable of killing virally-infected cells or tumor cells (Moretta et al., 2008), recent data indicate that NK cells also posses adaptive immune features (Cooper et al., 2009; Sun et al., 2009). Interaction between NK cells and dendritic cells (DC) is of particular interest, due to mutual modulation of cell function. For example, NK cells play an important role in controlling DC differentiation or in killing immature DC (Moretta et al., 2008). On the other hand, interferon alpha-producing pDC induce NK cytolytic activity (Colonna et al., 2004). The molecules responsible for "bringing these cells together" at sites of inflammation remain poorly defined. In this context it is of interest that NK cells have been shown to colocalize with pDC in oral lichen planus lesions in a chemerin-dependent manner (Parolini et al., 2007). Chemerin-mediated recruitment of pDC and NK cells might therefore represent a general mechanism to enable pDC and NK cell cross-regulation in diseased skin.

Recently, we and others have demonstrated that chemerin is present in psoriatic skin lesions, and likely plays a key role in regulating skin infiltration by pDC in psoriasis (Albanesi et al., 2009; Skrzeczynska-Moncznik et al., 2009). Although pDC are absent in normal skin, lesional skin from patients suffering from psoriasis vulgaris is characterized by substantial numbers of pDC. Infiltrating NK cells have also been described in psoriatic skin, although accumulation of NK cells was reported to be mediated by CXCL10, a ligand for CXCR3, and CCL5, a ligand for CCR5 (Ottaviani et al., 2006).

In this work we demonstrate that circulating NK cell subsets derived from psoriatic patients migrate to chemerin, suggesting that this chemoattractant contributes to NK cell migration to diseased skin. However, since NK cell infiltration does not always correlate with the presence of $\mathrm{pDC}$ in diseased skin, other chemoattractants may provide additive or alternative signals in recruiting these cells to affected skin.

\section{MATERIALS AND METHODS}

Chemoattractants. Recombinant human CXCL12 (SDF-1 $\alpha$ ) and recombinant human CXCL10 (IP-10) were purchased from R\&D Systems. StaphopainB (SspB) activated chemerin was prepared as described previously (Kulig et al., 2007). Briefly, recombinant human pro-chemerin, expressed in in- sect cells was treated with $S$. aureus-derived cysteine protease $\mathrm{SspB}$ at a 100:1 pro-chemerin:active $\mathrm{SspB}$ molar ratio at $37^{\circ} \mathrm{C}$ in a buffer containing $50 \mathrm{mM}$ Hepes, $100 \mathrm{mM} \mathrm{NaCl}, \mathrm{pH}$ 7.2. The 16155.4 Da SspBmediated cleavage product, further referred to as chemerin/SspB was purified by HPLC and used for cell migration experiments. Since SspB treatment of pro-chemerin generates a potent chemoattractant (Kulig et al., 2007), chemerin/SspB was used in all experiments as a bioactive form of this molecule.

Patients. All human studies were performed in accordance with guidelines established by the Jagiellonian University Institutional Bioethics Committee under approved protocols. Declaration of Helsinki protocols were followed. A total of 16 psoriasis patients (age $36.7 \pm 15.1$ years; F: 7, M: 9), 14 AD patients (age $28.9 \pm 6.9$ years; F: 6, M: 8 ) and 12 healthy individuals (age $33.3 \pm 10$ years; F: 5, M: 7) were enrolled into these studies. The severity of the psoriasis skin lesions was assessed according to the Psoriasis Area Severity Index score (PASI) (minimum point 0 , maximum point 72 ) and ranged from 9.6 to 34.6 (mean \pm S.D., 21.4 \pm 8.1 ). The AD patients were diagnosed according to the criteria defined by Hanifin and Rajka (1993). Disease severity in AD was measured according to the Severity Scoring of Atopic Dermatitis (SCORAD) (minimum point 0 , maximum point 100) and ranged from 12 to 82 (mean \pm S.D., $42.1 \pm 19.3$ ). Patients on UV therapy, systemic or local corticosteroid treatment, or with viral infections were excluded from the studies. Healthy control subjects had no clinical signs of dermatologic or allergic diseases.

Flow cytometry analysis. Human blood was collected and peripheral blood mononuclear cells (PBMC) were harvested following LSM1077 (PAA Laboratories) gradient separation. Cells were blocked with 50-80\% autologous plasma and then stained for flow cytometry using mAbs to CD56, CD16 and CD3 (reagents from BD Pharmingen eBioscience, Beckman Coulter and Dako). Stained cells were analyzed on an LSRII flow cytometer (Becton Dickinson).

NK cell chemotaxis assay. Blood was collected from psoriasis and $\mathrm{AD}$ patients as well as normal individuals, and PBMC were harvested as described above. The chemotaxis assay was performed using $5-\mu \mathrm{m}$ pore Transwell inserts. PBMC $\left(0.4-0.5 \times 10^{6}\right)$ in $100 \mu \mathrm{l}$ were added to the top wells, and various chemoattractants were added to the bottom wells in a $600 \mu \mathrm{l}$ volume. Migration was assayed for $2 \mathrm{~h}$ at $37^{\circ} \mathrm{C}$, then the inserts were removed, and the cells that had migrated through the filter to the lower chamber were harvested, stained with anti-CD16FITC, anti-CD56PE, and analyzed by flow cytometry. The number of gated NK cell subsets defined as $\mathrm{CD} 56^{\text {hi }} \mathrm{CD} 16-$ or $\mathrm{CD} 56^{\text {low }} \mathrm{CD} 6^{+}$subsets counted in $60 \mathrm{~s}$ was used as the migration output. 
Immunocytochemistry. Frozen $8-\mu \mathrm{m}$ sections were prepared from skin biopsies. Sections were fixed in acetone, blocked with mouse IgG and stained with the following directly conjugated antibodies; APC-anti-CD3 (BD Pharmingen) and PEanti-CD56 (Biolegend) or PE-anti-BDCA2 (Miltenyi Biotech).

Statistical analysis. For statistical evaluation, two-tailed Student's $t$-test was performed.

\section{RESULTS}

Psoriasis and atopic dermatitis (AD) represent chronically relapsing skin diseases with skin-infiltrating leukocytes playing a crucial role in the initiation and maintenance of both disorders (Leung et al., 2004; Nestle et al., 2005). However, whereas autoimmune psoriasis is characterized by substantial numbers of skin-infiltrating pDC that significantly contribute to the pathogenesis of the disease (Nestle et al., 2005), few pDC are present in skin lesions of AD patients (Wollenberg et al., 2002). Notably, the percentage of circulating pDC is lower in psoriasis patients compared to healthy individuals, whereas a higher frequency of $\mathrm{pDC}$ is noted in $\mathrm{AD}$ patients, suggesting that $\mathrm{pDC}$ leaving the circulation could account for the lower percentage of these cells among circulating leukocytes in patients suffering from psoriasis (Skrzeczynska-Moncznik et al., 2009). To examine whether NK cells follow a similar frequency pattern, we analyzed NK cell subsets derived from the blood of psoriasis and $\mathrm{AD}$ patients as well as normal donors by flow cytometry. Two subsets of circulating NK cells have been reported; CD$56^{\text {low }} \mathrm{CD} 16^{+} \mathrm{NK}$ effector cells, and CD56 ${ }^{\text {hi }} \mathrm{CD} 16^{-} \mathrm{NK}$ cells, characterized by limited cytotoxic function but a pronounced immunoregulatory phenotype (Ottaviani et al., 2006; Parolini et al., 2007). As shown in Fig. 1, similar percentages of CD56 ${ }^{\text {hi }} \mathrm{CD} 16^{-}$NK cells were found in normal individuals as well as psoriasis and $\mathrm{AD}$ patients. In contrast, the percentage of circulating CD56 low $\mathrm{CD}^{2} 6^{+} \mathrm{NK}$ cells was lower in $\mathrm{AD}$, but not psoriasis patients, compared to healthy individuals. The reduced frequency of NK cells in patients suffering from $\mathrm{AD}$ is in agreement with a previous report (Katsuta et al., 2006). Taken together, these data suggest that the recruitment of $\mathrm{pDC}$ and NK cell is regulated by different mechanisms.

To assess functional migratory responses of NK cells, we performed in vitro transwell chemotaxis assays on freshly isolated PBMC from normal, psoriasis, and AD patients, and stained for NK cell subsets after migration. The migratory response of NK cells to CXCL12, CXCL10 and chemerin was analyzed. All three chemoattractants have been shown before to support pDC migration to diseased skin

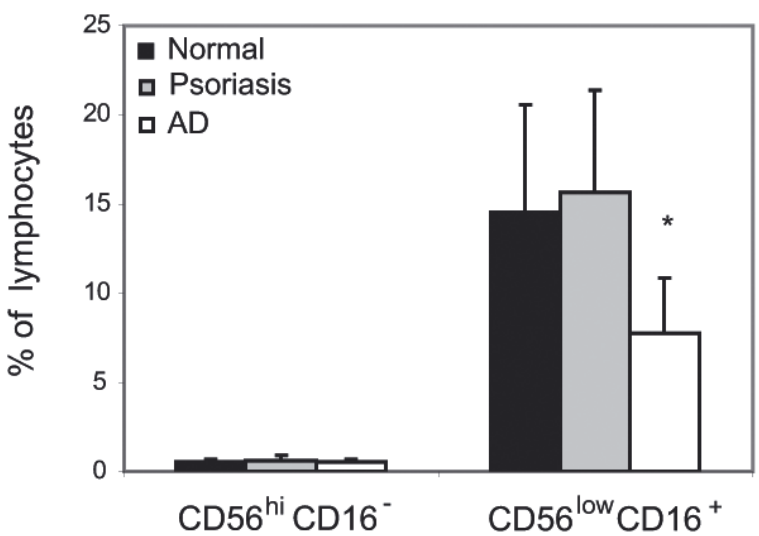

Figure 1. Circulating NK cells are present at a lower frequency in $\mathrm{AD}$ patients compared with psoriasis patients and normal donors.

PBMC were isolated from the blood of indicated donors and stained for NK cells using anti- CD56 and CD16 mAbs. The cells were then analyzed by flow cytometry. Data are shown as percentage of NK cell subsets among the cells falling in a lymphocyte light scatter gate (mean \pm S.D. for the indicated number of donors; normal $n=6$; psoriasis $n=10 ; A D n=8$ ). Statistically significant difference between normal and AD NK cell levels is indicated by asterisk $\left({ }^{*} P<0.05\right.$; Student's $t$-test).

(Vanbervliet et al., 2003; Albanesi et al., 2009; Skrzeczynska-Moncznik et al., 2009). Whereas the homeostatic chemokine CXCL12 and the inducible pro-inflammatory chemokine CXCL10 are present in both psoriatic and $\mathrm{AD}$ skin, active chemerin appears to be primarily associated with psoriasis (Albanesi et al., 2009; Skrzeczynska-Moncznik et al., 2009). In addition to $\mathrm{pDC}$, NK cells have been reported to express CMKLR1 (Vermi et al., 2005; Zabel et al., 2005b; 2006b; Parolini et al., 2007), suggesting that, similarly to $\mathrm{pDC}$, they might be recruited via chemerin activation in skin disorders. We first determined whether circulating NK cells derived from psoriasis patients migrated more efficiently to chemerin compared with normal controls. Of the two NK cell subsets, only the $\mathrm{CD} 56^{\text {low }} \mathrm{CD} 16^{+} \mathrm{NK}$ cells migrated to chemerin (Fig. 2), in agreement with previous studies demonstrating selective expression of functional CMKLR1 by CD56 ${ }^{\text {low }}$ CD $16^{+}$NK cells (Parolini et al., 2007). However, there were no significant differences in $\mathrm{CD}_{5} 6^{\text {low }} \mathrm{CD} 16^{+} \mathrm{NK}$ cell migration to chemerin among the patient groups (Fig. 2A). CD$56^{\text {low }} \mathrm{CD} 16^{+} \mathrm{NK}$ cells from all groups also responded with similar migration efficiency to CXCL12 (Fig. $2 \mathrm{~A})$. The CD56 hi CD16- NK cell subset, although unresponsive to chemerin, migrated efficiently to CXCL12 (Fig. 2B). CXCL10 was a more potent chemoattractant for CD56 hi CD16- NK cells than for CD$56^{\text {low }} \mathrm{CD} 16^{+} \mathrm{NK}$ cells, perhaps reflecting preferential expression of $\mathrm{CXCR} 3$ on the $\mathrm{CD} 16^{-}$subset (Ottaviani et al., 2006). A combination of CXCL12 and CXCL10 
A
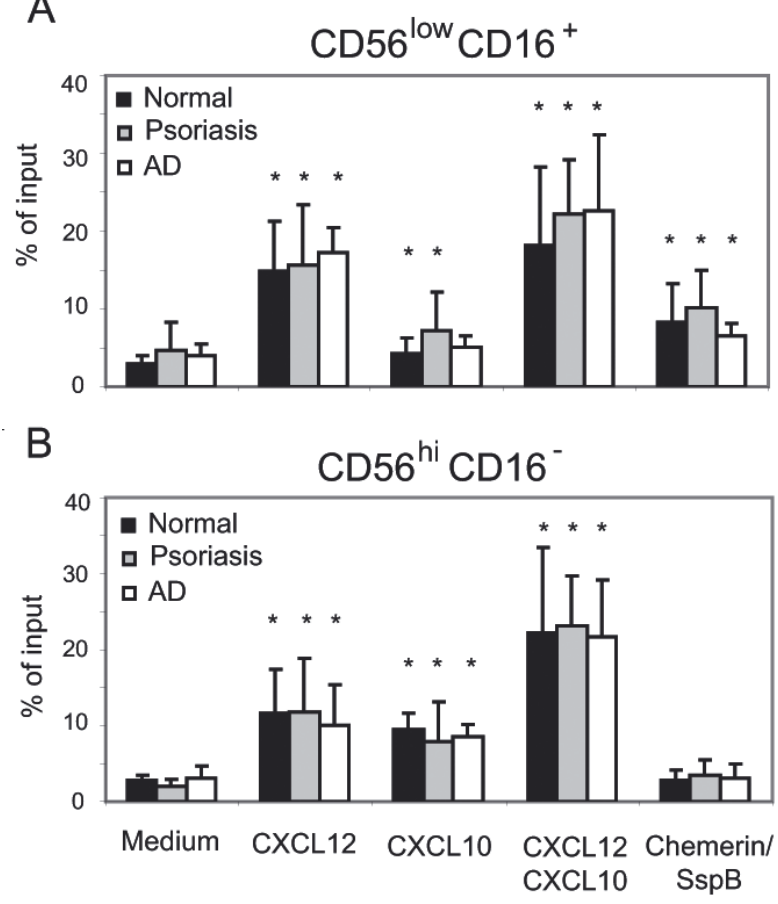

Figure 2. CD56 ${ }^{\text {low }} \mathrm{CD} 16^{+}$blood NK cells from normal donors as well as psoriasis and $\mathrm{AD}$ patients respond to active chemerin.

Total PBMC were tested in transwell chemotaxis, and the migrated cells were stained for CD56 and CD16 to define two circulating NK cell subsets, CD56 ${ }^{\text {low }} \mathrm{CD} 6^{+}$(A) and $\mathrm{CD}_{56}{ }^{\text {hi }} \mathrm{CD}_{16}{ }^{-}(\mathbf{B})$. The following chemoattractants were used as indicated: CXCL12 (10 nM), CXCL10 (115 nM) and chemerin/SspB (50 pM). Migration to chemotaxis medium served as a negative control (Medium). NK cell subset migration is displayed as the mean of six independent donors in each group \pm S.D. Statistically significant differences in the migration to the negative control (chemotaxis medium) vs. various chemoattractants in pairwise comparisons within patient groups was determined by Student's $t$-test $(P<0.05)$ and indicated by *

A

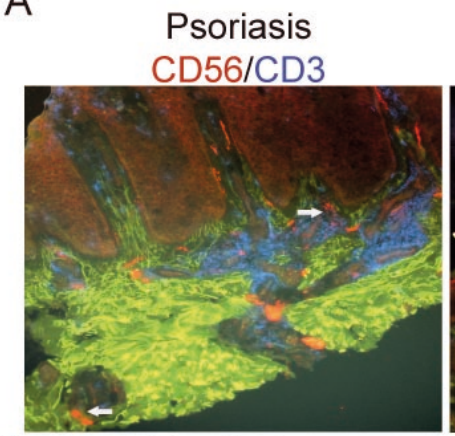

Psoriasis CD56/CD3

B

Psoriasis

CD56/CD3

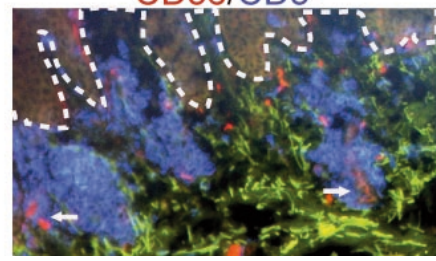

triggered the most robust chemotactic response in both NK cell subsets (Fig. 2). Taken together, these findings confirm a differential pattern of chemotactic responses by NK cell subsets but indicate similar chemotactic properties of NK cell subsets among the donor groups.

Previous immunohistochemical data suggest that NK cells are recruited to psoriasis and AD skin (Buentke et al., 2002; Ottaviani et al., 2006). In agreement with those studies, we found NK cells (CD56 ${ }^{+} \mathrm{CD}^{-}$cells of lymphocyte morphology) in the dermis of lesional skin from psoriasis and AD patients (Fig. 3A). NK cells were present throughout the $\mathrm{T}$ cell-rich infiltrate in the dermis, although small clusters of NK cells essentially devoid of $\mathrm{T}$ cells were also observed in inflamed AD skin (Fig. 3A). The same skin biopsies failed to stain for pDC (not shown), suggesting different recruitment pathways for NK cells and pDC. Since infiltration of psoriatic skin by pDC appears to be associated only with early phases of developing skin lesions (Albanesi et al., 2009), we also analyzed biopsies of pDC-infiltrated psoriatic skin for NK cells. As demonstrated in Fig. 3B, pDC were found in the dermis and the dermal papillae, in the latter case without obvious co-localization of $\mathrm{T}$ cells. In contrast, NK cells were primarily associated with $\mathrm{T}$ cell infiltrates in the dermis. Moreover, the distribution of NK cells was similar in pDC-positive and pDC-negative skin biopsies (Fig. $3 \mathrm{~A}$ and $\mathrm{B}$ ), suggesting that NK cell trafficking to psoriatic skin is not paralleled by pDC recruitment.

\section{DISCUSSION}

Psoriasis is one of the most common autoimmune disorders in humans and recent findings

Figure 3. NK cell skin infiltration does not correlate with pDC influx.

Frozen sections of skin biopsies from indicated patients were stained to detect $\mathrm{T}$ cells $\left(\mathrm{CD}^{+}\right.$, blue), $\mathrm{NK}$ cells $\left(\mathrm{CD}^{+} 6^{+}\right.$, red; $\left.\mathrm{CD}^{-}\right)$or $\mathrm{pDC}$ $\left(\mathrm{BDCA}^{+}\right.$, red). Autofluorescent components of extracellular matrix are shown in green. The slides were examined by fluorescence microscopy. Original magnification: $\times 10$. Dotted lines in $B$ indicate location of epidermis. Arrows indicate NK cells (upper panel and lower panel, left) and pDC (lower panel, right). Data shown in $B$ represent serial sections. Data are representative of three different donors in each staining combination. 
strongly implicate activated $\mathrm{pDC}$ in this pathologic condition (Nestle et al., 2005; Lande et al., 2007). Interestingly, both pDC and NK cells constitute the inflammatory infiltrate in psoriasis, suggesting possible crosstalk between these cells in the initiation and/or maintenance of psoriatic inflammation. The mutual interaction of pDC with NK cells has been reported to profoundly influence activation status of both types of cells, leading on one hand to the augmentation of NK cell cytolytic activity by pDC, and on the other hand, to the induction of pDC maturation by activated NK cells (Gerosa et al., 2005). Therefore, reciprocal contact between $\mathrm{pDC}$ and NK cells would be predicted to be of important consequence for the development of psoriatic lesions and a better understanding of the rules that govern the pDC-NK relationship will help develop new approaches for the diagnosis and/or treatment of psoriasis.

Since circulating pDC and NK cells both express CXCR3 and CMKLR1, we reasoned that their respective ligands, CXCL10 and chemerin, might contribute to either pDC or NK cell influx to psoriatic skin, enabling local interactions between these cells in psoriatic plaques. Chemerin appears to cooperate with CXCL10 in directing pDC to skin in psoriasis (Skrzeczynska-Moncznik et al., 2009). Notably, while $\mathrm{CD} 56^{\text {hi }} \mathrm{CD} 16^{-} \mathrm{NK}$ cell infiltrates have been reported in psoriatic skin, CD16 ${ }^{+} \mathrm{NK}$ cells have not been detected (Ottaviani et al., 2006). Since circulating CD56 ${ }^{\text {hi }} \mathrm{CD}^{-} 6^{-} \mathrm{NK}$ cells from psoriasis patients do not migrate to chemerin (while CD16 ${ }^{+} \mathrm{NK}$ cells do) (Fig. 2), this implies that chemerin-independent mechanisms likely guide the recruitment of CD56 ${ }^{\mathrm{hi}}$ CD16- NK cells to psoriatic skin, and that the regulation of $\mathrm{CD}_{16}{ }^{+} \mathrm{NK}$ cell recruitment is more complex than simply by chemerin: CMKLR1 interactions. Other chemokines might provide alternative or additional signals to regulate NK cell infiltration of diseased skin, including CCL5, CCL4 or CXCL8 (Leung et al., 2004; Ottaviani et al., 2006; Parolini et al., 2007).

Chemerin has been shown to play a role in the co-localization of NK cells and pDC in oral lichen planus lesions (Parolini et al., 2007), suggesting that the accumulation of both NK cells and pDC observed in other skin diseases might depend on this chemoattractant. However, the results of this work together with previous reports (Parolini et al., 2007; Albanesi et al., 2009; Skrzeczynska-Moncznik et al., 2009) suggest that, despite being chemerin-responsive, pDC and NK cells follow distinct trafficking patterns in skin disorders. First, the differences in the frequency of circulating $\mathrm{pDC}$ and NK do not similarly reflect disease-associated variations in $\mathrm{pDC}$ and NK cell migration to local sites. Furthermore, NK cells infiltrate either psoriasis or AD skin, even though AD skin appears to be largely chemerin- negative (Skrzeczynska-Moncznik et al., 2009) and pDC are thought to be generally absent in AD skin lesions (Wollenberg et al., 2002). Moreover, psoriatic skin infiltration by NK cells does not correlate with pDC recruitment into skin, since NK cells mixed with $\mathrm{T}$ cells can be found in psoriatic skin despite the absence of pDC. Finally, pDC-positive biopsies of lesional psoriatic plaques that are likely to contain chemotactively active chemerin (Albanesi et al., 2009; Skrzeczynska-Moncznik et al., 2009) do not show obvious co-localization of NK cells and pDC.

Taken together these data suggest at least partially distinctive homing properties of $\mathrm{pDC}$ and NK cells to diseased skin and implicate other chemoattractants that may play additive effects in recruiting these leukocytes to inflamed skin.

\section{Acknowledgements}

We thank Teresa Złotogórska for help with immunohistochemistry.

This work was supported in part by grants from the Ministry of Scientific Research, Poland SPUB3088 (to J.C.), by Fogarty International Research Collaborative Award R03TW007174-01 (to E.C.B. and J.C.) and by grant from EU 6th FP project SP6MTKDCT-2006-042586 (to J.C.) and NIH AI-59635 to E.C.B.

\section{REFERENCES}

Albanesi C, Scarponi C, Pallotta S, Daniele R, Bosisio D, Madonna S, Fortugno P, Gonzalvo-Feo S, Franssen JD, Parmentier M, De Pità O, Girolomoni G, Sozzani S (2009) Chemerin expression marks early psoriatic skin lesions and correlates with plasmacytoid dendritic cell recruitment. J Exp Med 206: 249-258.

Buentke E, Heffler LC, Wilson JL, Wallin RP, Löfman C, Chambers BJ, Ljunggren HG, Scheynius A (2002) Natural killer and dendritic cell contact in lesional atopic dermatitis skin - Malassezia-influenced cell interaction. J Invest Dermatol 119: 850-857.

Cash JL, Hart R, Russ A, Dixon JP, Colledge WH, Doran J, Hendrick AG, Carlton MB, Greaves DR (2008) Synthetic chemerin-derived peptides suppress inflammation through ChemR23. J Exp Med 205: 767-775.

Colonna M, Trinchieri G, Liu YJ (2004) Plasmacytoid dendritic cells in immunity. Nat Immunol 5: 1219-1226.

Cooper MA, Elliott JM, Keyel PA, Yang L, Carrero JA, Yokoyama WM (2009) Cytokine-induced memory-like natural killer cells. Proc Natl Acad Sci USA 106: 19151919.

Du XY, Zabel BA, Myles T, Allen SJ, Handel TM, Lee PP, Butcher EC, Leung LL (2009) Regulation of chemerin bioactivity by plasma carboxypeptidase N, carboxypeptidase B (activated thrombin-activable fibrinolysis inhibitor), and platelets. J Biol Chem 284: 751-758.

Gerosa F, Gobbi A, Zorzi P, Burg S, Briere F, Carra G, Trinchieri G (2005) The reciprocal interaction of NK cells with plasmacytoid or myeloid dendritic cells profoundly affects innate resistance functions. I Immunol 174: 727-734. 
Goralski KB, McCarthy TC, Hanniman EA, Zabel BA, Butcher EC, Parlee SD, Muruganandan S, Sinal CJ (2007) Chemerin, a novel adipokine that regulates adipogenesis and adipocyte metabolism. I Biol Chem 282: 28175-28188.

Katsuta M, Takigawa Y, Kimishima M, Inaoka M, Takahashi R, Shiohara T (2006) NK cells and gamma delta+ $\mathrm{T}$ cells are phenotypically and functionally defective due to preferential apoptosis in patients with atopic dermatitis. J Immunol 176: 7736-7744.

Kulig P, Zabel BA, Dubin G, Allen SJ, Ohyama T, Potempa J, Handel TM, Butcher EC, Cichy J (2007) Staphylococcus aureus-derived staphopain B, a potent cysteine protease activator of plasma chemerin. J Immunol 178: 3713-3720.

Lande R, Gregorio J, Facchinetti V, Chatterjee B, Wang $\mathrm{YH}$, Homey B, Cao W, Wang YH, Su B, Nestle FO, Zal T, Mellman I, Schröder JM, Liu YJ, Gilliet M (2007) Plasmacytoid dendritic cells sense self-DNA coupled with antimicrobial peptide. Nature 449: 564-569.

Leung DY, Boguniewicz M, Howell MD, Nomura I, Hamid QA (2004) New insights into atopic dermatitis. J Clin Invest 113: 651-7

Moretta A, Marcenaro E, Parolini S, Ferlazzo G, Moretta L (2008) NK cells at the interface between innate and adaptive immunity. Cell Death Differ 15: 226-233.

Nagpal S, Patel S, Jacobe H, DiSepio D, Ghosn C, Malhotra M, Teng M, Duvic M, Chandraratna RA (1997) Tazarotene-induced gene 2 (TIG2), a novel retinoid-responsive gene in skin. J Invest Dermatol 109: 91-95.

Nestle FO, Conrad C, Tun-Kyi A, Homey B, Gombert M, Boyman O, Burg G, Liu YJ, Gilliet M (2005) Plasmacytoid predendritic cells initiate psoriasis through interferon-alpha production. J Exp Med 202: 135-143.

Ottaviani C, Nasorri F, Bedini C, de Pita O, Girolomoni G, Cavani A (2006) CD56brightCD16(-) NK cells accumulate in psoriatic skin in response to CXCL10 and CCL5 and exacerbate skin inflammation. Eur J Immunol 36: 118-128.

Parolini S, Santoro A, Marcenaro E, Luini W, Massardi L, Facchetti F, Communi D, Parmentier M, Majorana A, Sironi M, Tabellini G, Moretta A, Sozzani S (2007) The role of chemerin in the colocalization of NK and dendritic cell subsets into inflamed tissues. Blood 109: 3625-3632.

Severity scoring of atopic dermatitis: the SCORAD index (1993) Consensus Report of the European Task Force on Atopic Dermatitis. Dermatology 186: 23-31.

Skrzeczyńska-Moncznik J, Wawro K, Stefańska A, Oleszycka E, Kulig P, Zabel BA, Sułkowski M, KapińskaMrowiecka M, Czubak-Macugowska M, Butcher EC, Cichy J (2009) Potential role of chemerin in recruitment of plasmacytoid dendritic cells to diseased skin. Biochem Biophys Res Commun 380: 323-327.

Sun JC, Beilke JN, Lanier LL (2009) Adaptive immune features of natural killer cells. Nature 457: 557-561.

Vanbervliet B, Bendriss-Vermare N, Massacrier C, Homey B, de Bouteiller O, Brière F, Trinchieri G, Caux C (2003) The inducible CXCR3 ligands control plasmacytoid dendritic cell responsiveness to the constitutive chemokine stromal cell-derived factor 1 (SDF-1)/CXCL12. J Exp Med 198: 823-830.

Vermi W, Riboldi E, Wittamer V, Gentili F, Luini W, Marrelli S, Vecchi A, Franssen JD, Communi D, Massardi L, Sironi M, Mantovani A, Parmentier M, Facchetti F, Sozzani S (2005) Role of ChemR23 in directing the migration of myeloid and plasmacytoid dendritic cells to lymphoid organs and inflamed skin. J Exp Med 201: 509-515.

Wittamer V, Bondue B, Guillabert A, Vassart G, Parmentier M, Communi D (2005) Neutrophil-mediated maturation of chemerin: a link between innate and adaptive immunity. J Immunol 175: 487-493.

Wittamer V, Franssen JD, Vulcano M, Mirjolet JF, Le Poul E, Migeotte I, Brézillon S, Tyldesley R, Blanpain C, Detheux M, Mantovani A, Sozzani S, Vassart G, Parmentier M, Communi D (2003) Specific recruitment of antigen-presenting cells by chemerin, a novel processed ligand from human inflammatory fluids. J Exp Med 198: 977-985.

Wollenberg A, Wagner M, Günther S, Towarowski A, Tuma E, Moderer M, Rothenfusser S, Wetzel S, Endres S, Hartmann G (2002) Plasmacytoid dendritic cells: a new cutaneous dendritic cell subset with distinct role in inflammatory skin diseases. J Invest Dermatol 119: 1096-1102.

Zabel BA, Allen SJ, Kulig P, Allen JA, Cichy J, Handel TM, Butcher EC (2005a) Chemerin activation by serine proteases of the coagulation, fibrinolytic, and inflammatory cascades. J Biol Chem 280: 34661-34666.

Zabel BA, Silverio AM, Butcher EC (2005b) Chemokine-like receptor 1 expression and chemerin-directed chemotaxis distinguish plasmacytoid from myeloid dendritic cells in human blood. J Immunol 174: 244-251.

Zabel BA, Ohyama T, Zuniga L, Kim JY, Johnston B, Allen SJ, Guido DG, Handel TM, Butcher EC (2006a) Chemokine-like receptor 1 expression by macrophages in vivo: regulation by TGF- $\beta$ and TLR ligands. Exp Hematol 34: 1106-1114.

Zabel BA, Zuniga L, Ohyama T, Allen SJ, Cichy J, Handel TM, Butcher EC (2006b) Chemoattractants, extracellular proteases, and the integrated host defense response. Exp Hematol 34: 1021-1032. 